\title{
Die FMH-Ärztestatistik als Instrument der Bedarfsplanung?
}

\author{
Zur FMH-Ärztestatistik siehe \\ auch das Editorial von \\ Dr. med. Daniel Herren, \\ Mitglied des Zentralvorstands \\ der FMH.
}

\section{Das sagen die Wissenschaftlerin, der Politiker und der Hausarzt}

A: Weshalb ist ein aussagekräftiges Zahlenmaterial für das schweizerische Gesundheitswesen nötig? B: Warum ist eine solide Datenbasis für die Ärzteschaft wichtig? C: Wie hoch schätzen Sie die Relevanz der FMH-Ärztestatistik als Instrument der Bedarfsplanung ein?

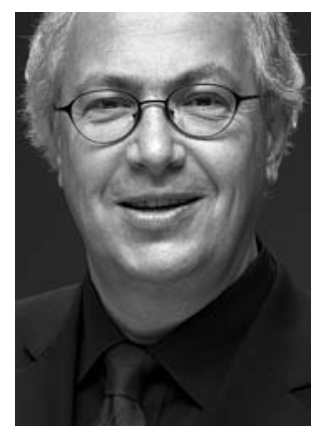

Thomas Burgener Staatsrat Kanton Wallis, Vorsteher des Departements für Gesundheit, Sozialwesen und Energie

A: Bis zum Ende der Zulassungsbeschränkung (Ende 2009) muss eine neue Reglementierung für die Zulassung der Leistungserbringer erstellt werden. Die Krankenversicherer befürworten die Einführung der Vertragsfreiheit, ich meinerseits lehne die Aufhebung des Kontrahierungszwangs ab: Die Macht der Kassen darf nicht weiter gestärkt werden. Die Kantone und die FMH haben einen alternativen Vorschlag ausgearbeitet: Das Prinzip des Kontrahierungszwangs wird aufrechterhalten, die Kantone haben jedoch die Möglichkeit, im Falle eines Ärztemangels oder -überschusses dirigierend einzugreifen. Es gilt nun, die eidgenössischen Räte zu überzeugen, die anscheinend immer noch ein auf der Vertragsfreiheit basierendes System befürworten.

Unabhängig von der Reglementierung: Zuverlässige und aussagekräftige statistische Daten sind notwendig, um der Bevölkerung ein ausreichendes Pflegeangebot zu garantieren.

B: Die Erfahrung mit der Zulassungsbeschränkung der Leistungserbringer zeigte: Es ist sehr wichtig, solide statistische Daten zu erheben, um das Angebot sowohl für den Patienten als auch für den Leistungserbringer in einer befriedigenden Weise regulieren zu können. Das Arbeitspensum, die tatsächlich ausgeübten Disziplinen und die Qualität der Leistungen wurden bisher nicht erfasst. Zudem übt der Aufbau von Gruppenpraxen Druck auf die Reglementierung aus.

In Zukunft werden ausreichend präzise Daten notwendig sein, damit das Dosierungssystem für das Angebot verbessert und somit in jeder Region eine Unter- oder Überversorgung vermieden werden kann.
C: Die FMH-Ärztestatistik ist ein sehr aussagekräftiges Instrument und für die kantonalen Gesundheitsbehörden von grosser Wichtigkeit. Sie ermöglicht die laufende Erfassung der Anzahl praktizierender Ärzte pro Kanton und Disziplin. Sie dient zudem als Basis für verschiedene Studien, die das medizinische Angebot unter Berücksichtigung des Beschäftigungsgrades und der effektiv ausgeübten Disziplinen messen.

Im Jahre 2000 haben wir in unserem Kanton das Walliser Gesundheitsobservatorium eingeführt. Dieses Gesundheitsinformationssystem hat die Aufgabe, die Gesamtheit der aktuellen Daten (stationärer und ambulanter Bereich) zu erfassen. Dafür ist die FMH-Statistik eine unabdingbare Datenquelle.

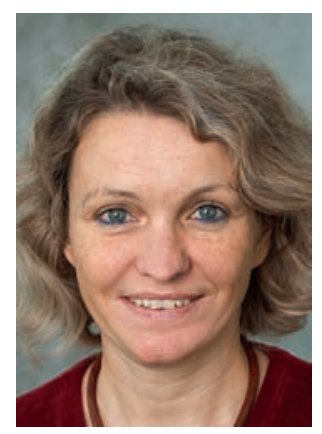

Hélène Jaccard Ruedin Projektleiterin, Obsan

A: Der Zugang zur Gesundheitsversorgung ist ein Grundrecht aller Bürgerinnen und Bürger. Dies erfordert eine gewisse Planung der Infrastruktur und der Ressourcen. Zur Gewährleistung der Finanzierung ist es ausserdem notwendig, dass bestimmte Steuerungsmassnahmen umgesetzt werden. In diesem Zusammenhang sind auf verschiedenen Ebenen qualitativ hochwertige Daten erforderlich:

- Indem die Bedürfnisse der Bevölkerung und der Umfang und die Art der Ressourcen abgeklärt werden, die für die Befriedigung dieser Bedürfnisse benötigt werden, erleichtern diese Daten die Planung, die Steuerung und die Organisation des Gesundheitswesens, so dass die Verfügbarkeit der entsprechenden Leistungen gewährleistet werden kann.

- Indem diese Daten Angaben zur Zusammensetzung der Gesundheitskosten liefern, tragen sie zum Verständnis der Dynamik des Systems bei. 
Und da diese Daten die Entwicklung des Gesundheitssystems beschreiben, ermöglichen sie die Beurteilung der Auswirkungen der getroffenen Steuerungsmassnahmen.

B: Was die Ärzteschaft anbelangt, hängt das Interesse an qualitativ hochwertigen Daten vom Bereich ab, den diese Daten beschreiben:

- Die Daten, die die Bedürfnisse der Bevölkerung und deren Entwicklung beschreiben, tragen zur epidemiologischen Forschung bei. Sie ermöglichen Anpassungen der ärztlichen Versorgung und eine Beurteilung der Auswirkungen von medizinischen Leistungen, wie beispielsweise von Präventionsmassnahmen.

- Die Daten, die die in Anspruch genommenen bzw. erbrachten Leistungen beschreiben, liefern Informationen zu den Ressourcen, die zur Befriedigung eines bestimmten Bedürfnisses eingesetzt werden müssen. Sie sind ein grundlegendes Instrument für die medizinische Forschung, die sich mit der Qualität der Gesundheitsversorgung befasst.

- In einem anderen Bereich wurden die Daten, die die verfügbaren Ressourcen beschreiben, für die Regulierung der Bestände verwendet. In diesem Fall tragen qualitativ hochwertige Daten dazu bei, dass Auslegungsfehler vermieden werden.

C: Mit der neuen FMH-Statistik wird die Ärzteschaft detaillierter beschrieben. Die Ärztebestände werden nach Arbeitsort, Beschäftigungsgrad oder nach bestimmten grundlegenden Tätigkeiten unterschieden. Damit zeichnet die Ärztestatistik ein realistischeres Bild von den verschiedenen Arten von ärztlichen Leistungen, die auf regionaler Ebene zur Verfügung stehen. Indem die Entwicklung des Angebots verfolgt wird, können die Auswirkungen der Tendenzen, die sich innerhalb der Ärzteschaft abzeichnen, und von politischen Massnahmen, die als Anreiz oder zur Einschränkung getroffen werden, beurteilt werden.

Die Ärztestatistik erleichtert auch Prognosen hinsichtlich des künftigen Ärztebedarfs in den verschiedenen Bereichen.

Es ist jedoch darauf hinzuweisen, dass mit Hilfe dieser Statistik nur Prognosen, jedoch keine Planungen erstellt werden können. Für die letztere Aufgabe, die beispielsweise mit den angloamerikanischen «Health Systems» realisiert wird, sind viel detailliertere Daten erforderlich.

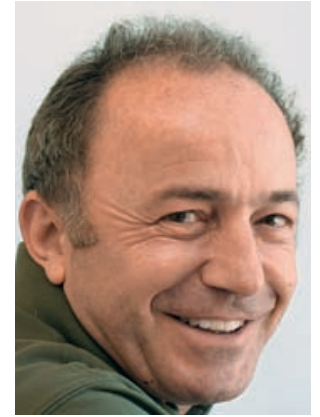

Franz Marty

Hausarzt in Chur, Facharzt für Allgemeinmedizin FMH

A: Aussagekräftige Zahlen:

- erlauben eine zuverlässige Dokumentation der Interessenfelder;

- helfen bei der Analyse von Fragestellungen;

- ermöglichen Standortbestimmungen;

- sind schliesslich die Grundlage jeder Planung.

Eine besondere Herausforderung - gerade im Gesundheitswesen - ist das Finden der wesentlichen Daten im Meer der Zahlen. Deshalb sind unabhängige, kostengünstige und robuste Indikatoren wichtig, die in einfacher Weise zu erheben sind.

B: Solide Daten sind in allen - beruflichen wie administrativen - Bereichen wichtig: In der täglichen Arbeit stützen wir uns auf präzise Labordaten, aggregieren unsere Abrechnungen (Leistungsdaten) zu einer nützlichen Datenbasis, und Lehre und Forschung liegen aussagekräftige Daten zugrunde.

Gerade im Bereich der Entschädigung für die erbrachten Leistungen zeigten die letzten Jahre, dass das ökonomische Überleben des selbständigen Hausarztes ohne solide dokumentierte Daten kaum gewährleistet werden kann (Trustcenter).

C: Die neue FMH-Ärztestatistik dokumentiert erstmalig die hausärztlich tätigen Ärzte. Durch die Erfassung des Arbeitspensums sind Rückschlüsse auf die hausärztliche Workforce möglich. Die Angaben zum Praxistyp werden ebenfalls erfasst (Einzel- oder Gruppenpraxis).

Der Aufwand für das einzelne Mitglied beschränkt sich auf ein Minimum, die Chance einer breiten Akzeptanz und Beteiligung bei der Datenerfassung ist somit gross. Die FMH-Ärztestatistik wird sicher zu einem relevanten, für die Hausarztmedizin stark verbesserten Instrument der Bedarfsabschätzung. Die Kenntnis der Workforce ist aber nur die eine Seite der Medaille, der «gesellschaftliche Bedarf» an Hausarztmedizin (neue Therapieangebote, Demographie) die andere und eine weitere zu erhebende Grösse. 